\title{
Analysis of a Video-Based Pain Monitoring System in Raspberry Pi
}

\author{
$1^{\text {st } J h o n a t a n ~ S o u z a ~}$ \\ Universidade Federal do Paraná \\ jhonatansouza@ufpr.br \\ Curitiba - Brasil
}

\author{
$2^{\text {nd }}$ Claudemir Casa \\ Universidade Federal do Paraná \\ claudemir.casa@ufpr.br \\ Curitiba - Brasil
}

\author{
$3^{\text {rd }}$ André Roberto Ortoncelli \\ Universidade Tecnológica do Paraná \\ ortoncelli@utfpr.br \\ Dois Vizinhos - Brasil
}

\begin{abstract}
This work presents an analysis of the efficiency and effectiveness of a Video-Based Pain Monitoring System running on a Raspberry - selected because it is a cheap device that can be easily carried around. The objective of the evaluated system is to allow the assessment of pain based on two characteristics: Heart Rate (HR) and facial expressions detected through the Facial Action Coding System (FACS). To measure HR an Eulerian Video Magnification (EVM) based method was implemented. EVM is one of the main current approaches to measure HR by Remote PhotoPlethysmoGraphy. FACS was used to detect pain intensity with the Prkachin and Solomon Pain Intensity (PSPI) equation which is one of the most used approaches to detect pain intensity based on facial features. To identify the PSPI value we trained a Regression Neural Network (RNN) with the UNBC-McMaster database. The experimental results demonstrate the strengths and limitations of the evaluated system.

Index Terms-Action Untis, Remote PhotoPlethysmoGraphy, Paint estimation, Low-cost system
\end{abstract}

\section{INTRODUCTION}

There are several definitions for pain in the literature. According to the Medical Dictionary by Farlex, pain is an unpleasant feeling that is conveyed to the brain by sensory neurons [1]. For the North American Nursing Diagnosis Association, pain is a state in which a person experiences and reports an uncomfortable sensation or severe discomfort [2]. A critical analysis of pain definitions can be found in [3].

Despite the different definitions, it is consensual that pain can vary widely in intensity, quality, duration, pathophysiological mechanisms, and meanings [3]. It is also a fact that if the pain is not effectively relieved, it has a detrimental effect on all aspects of quality of people life [4].

The assessment is one of the main difficulties related to the pain diagnosis, which is usually done by self-report. The main problem with this type of self-assessment is subjectivity. There are also groups of people for whom the use of self-reports may not be effective: people with dementia [5], neonates [6], [7], and unconscious patients [8].

Due to the limitations of self-reports, automatic pain detection techniques are extremely important. It is possible to highlight recent works that report this type of approach, commonly based on characteristics extracted from human actions [9] or physiological signs [10].

Concerning physiological signs, sensors in contact with human skin can be a problem in some situations, such as in people with as Pemphigus Vulgaris or with skin wounds [11],
[12]. It is also noteworthy that invasive monitoring means can increase the chances of infections in patients and even worsen their health status [13].

Means of measuring physiological signs without physical contact can improve the individuals' quality of life, enable a large-scale population screening, be more hygienic, reduce costs and the need for skilled labor in handling complex equipment, and bring more freedom to the patient [11], [14].

Recent works based on Computer Vision, automatically and non-invasively assess pain, based on different characteristics, such as facial expressions and movements [9], skin temperature detected by infrared cameras [15] and Heart Rate (HR) recognized by Remote PhotoPlethysmoGraphy (rPPG) [16].

Even with the current literature, there is still room for contributions, such as: improving prediction results, reducing equipment costs, and improvements in human-computer interfaces. These contributions are relevant to support new pain assessment approaches that can be proposed and used more frequently by a larger group of people.

In this context, the objective of this work is to conduct a case study to analyze the efficiency and effectiveness of a noninvasive video-based system for pain assessment performed on a Raspberry $\mathrm{Pi}$ - selected because it is cheap and small device that can be easily carried around. In the experiments, we compared the results obtained from the Raspberry Pi with two traditional computers.

The validated system aims to continuously detects two pieces of information from the person's faces: i) HR - with an rPPG method based on Eulerian Video Magnification (EVM), that is one of the main current approaches to measure HR by rPPG [17]; ii) and pain intensity, recognized by a Neural Regression Network (RNN) that detects pain intensity based on Prkachin and Solomon Pain Intensity (PSPI) [18] equation - which is based on the Facial Action Coding System (FACS) [19]. PSPI is one of the most used approaches to detect pain intensity based on facial features.

We chose these multimodal features, because the fusion between physiological signals and FACS has a positive impact on the accuracy of pain recognition compared to the use of only one modality [20].

In the experiments, we used two databases: i) UNBCMcMaster [21], which presents videos of faces, with frameby-frame FACS, scores of pain, sequence-level self-report, 
and observer measures; and ii) a database that we created to validate the HR detection algorithm.

The case study was evaluated in relation to two groups of characteristics: i) the accuracy of the results in relation to the ground truth; and ii) the amount of resources consumed in terms of time, usage of CPU/GPU, and RAM memory consumed.

Experimental results indicate that it is feasible to use a Raspberry Pi to identify pain intensity based on PSPI value, detected by an RNN from the face image. The results regarding the rPPG method are not so positive. If the videobased recognition of these two multimodal features (with the algorithms analyzed in this case study) were combined in a Raspberry Pi, it would be unfeasible to use in addition to that pain monitoring system, because of the processing volume, the device will restart to avoid warming up.

We hope that our study will contribute to the development of new algorithms that consume less computational resources and enable the use of the analyzed pain monitoring approach.

The remainder of this text is organized as follows. Section II presents theoretical aspects important to understand our work. The experiments are presented and analyzed in Section III. By the end, conclusions are in Section IV

\section{THEORETICAL FOUNDATION}

This section presents concepts relevant to the understanding of this work. Subsection II-A describes techniques used to measure the heart rate of people, with a focus on non-contact approaches. Subsection II-B presents the method that we use to remotely measure HR. Subsection II-C presents details of pain assessment based on Action Units.

\section{A. Heart Rate measurement}

One of the main automatic techniques to measure heart rate is Photoplethysmography (PPG). This technique uses photoplethysmography sensors to measure the amount of infrared light absorbed or reflected by the blood, which changes according to the blood volume [22].

The PPG signal can be extracted from different parts of the body, such as finger, arm, and face [23]. Finger oximeters are devices widely used these days for this purpose - we use this equipment to produce the ground truth of our experimental database.

Although there are consolidated techniques based on physical contact to measure HR (such as PPG), sensors in contact with skin can be a problem for some people with rare diseases [11], [12], beyond of increase the chances of infections in patients and even worsen their health status [13].

In this context, the development of sensors to estimate HR without skin contact is extremely important, as in addition to being more hygienic, this type of sensor can reduce costs, allow large-scale population screening, in addition to giving more freedom to the patient [11], [14].

Some authors explored video-based approaches to detecting HR. Eulerian Video Magnification (EVM) is one of the main techniques used [17]. Since the EVM was created [24], it has been explored for HR detection [25], [26], showing good results in recent research [27]-[29]. Due to these results, we chose an EVM-based method to measure HR in our experiments - these technique is detailed in the following Subsection.

\section{B. Heart Rate estimation based on EVM}

This subsection presents the method used in the experiments to estimate the HR of facial videos. This method is based on EVM - a technique that reveals low amplitude movements and amplifies image colors that are not perceptible to the naked eye. The amplification step reveals variations in redness as blood flows over the face, which makes it possible to measure the HR.

We explore the classic EVM method, which receives as input a sequence of adjacent frames in a video and applies a temporal and spatial amplification process to each frame: i) the original video is divided into a sequence of $n$ frames $(n=300$, in our experiments); ii) in each frame a process is applied to amplify colors, allowing the analysis of the blood volume variation; and iii) are generated shows spatiotemporal slices that represent the frames of the original video with amplified colors. Mode details on the classic EVM are in [24]. Figure 1 presents an example of EVM inputs and outputs considering $\mathrm{n}=4$.

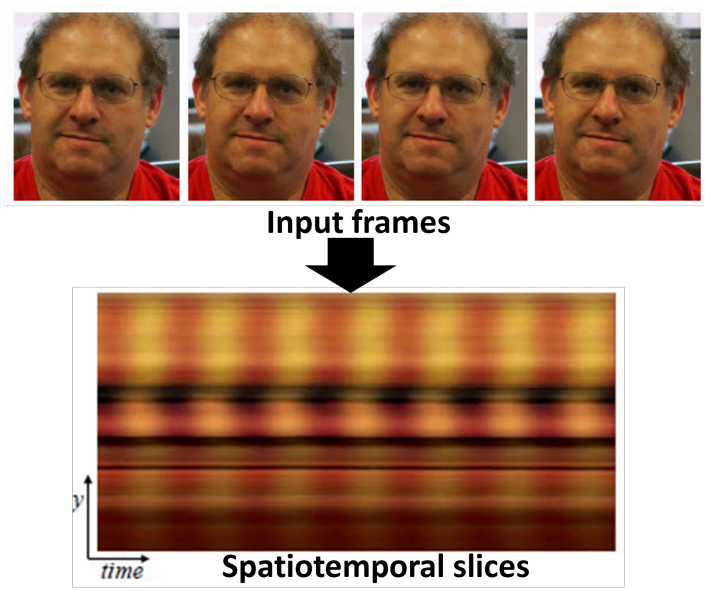

Fig. 1: EVM input and output - adapted from [24].

Our algorithm to measure HR by rPPG is based on five main steps: i) detection of 68 facial landmarks with the Dlib library [30]; ii) the landmarks are used to select two ROI's in areas of the face with large blood circulation [31] (Figure 2); iii) EVM [24] is applied in the ROI's of 300 adjacent frames (representing 10 seconds of video); iv) the band-pass filter is applied to remove low and high-frequency signals; v) the fast Fourier transform (FFT) [32] is applied and the HR is obtained with the highest peak.

\section{Pain Detection based on Facial Expression}

A Facial Expression (FE) is a movement of one or more muscles of the face, which can be a sign of emotion, but may 


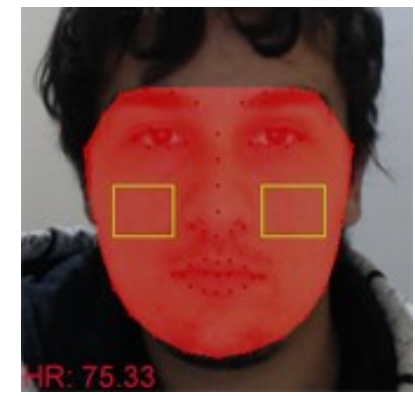

Fig. 2: ROI's used to detect HR in our method.

have other purposes, such as signaling the taking of turns, transmitting biometric information, expressing the intensity of mental effort [33], and indicating pain [18]. One of the main approaches to conceptualizing FE is the Facial Action Coding System (FACS). Based on the FACS, each FE can be defined as a set of individual facial muscle actions - called Action Unit's (AU's). Currently, there is a set of 44 AU's that was created by Carl-Herman Hjortsjö in 1970, and extended by Paul Ekman and Wallace Friesen in 2002 [19].

FACS has been explored to detect pain in facial expressions. The work of [18] is fundamental, as it proposed an equation - the Prkachin and Solomon Pain Intensity (PSPI), which measures pain with a linear combination of the intensity of the AUS on a 16-level scale (Equation 11. The PSPI is based on AU's 4, 6, 7, 9, 10, and 43 - Examples of these AU's can be found in [34].

$$
A U 4+\max (A U 6, A U 7)+\max (A U 9, A U 10)+A U 43
$$

It should be noted that the PSPI equation has been used with good results to estimate pain in recent works [9], [35], because of this, we select this approach to our case study.

\section{EXPERIMENTS}

This Section describes our experiments. Subsection III-A displays the database. The experimental configuration is detailed in Subsection III-B. The results are presented in Subsection III-C The analysis of the results is in the Subsection III-D

\section{A. Databases}

We used two databases in the case study:

- UNBC-McMaster database: for pain detection based on AU's with the PSPI equation (Equation 11), the UNBCMcMaster database [21] was used. These database focuses on videos of faces with people with shoulder pain, 48,398 FACS annotated in 200 videos. In addition to facial expressions, the database also has pain frame-byframe scores and sequence-level self-report and observer measures. This database is used in several recent works [20], [35].

- Our database:To evaluate the HR measurement algorithm (Section $\Pi \mathrm{I}-\mathrm{A}$ we produce a specific database. Our database is made up of face videos of 30 participants (22 men and 8 women). During the experiment, subjects stood in front of the camera (at a distance of one meter). All videos were collected in a controlled environment with controlled lighting.

For each person, an average of one minute of video was collected, referring to the time that the Raspberry hardware (described in the next Subsection) was able to function normally, without restarting due to overheating. Our database ground truth was produced with a pulse oximeter (model CMS50E from ContecMedical) - used by the participants when the videos were collected. The oximeter data is synchronized with the video frames.

In experiments carried out to detect HR by PPG, it is important to consider that these methods have worse results for darker skin colors. The motive for this is that dark skin contains more melanin and absorbs more green light than lighter skin. In our database, the skin color of the 30 participants are between types II and V of the Fitzpatrick Scale [36] (Figure 3).

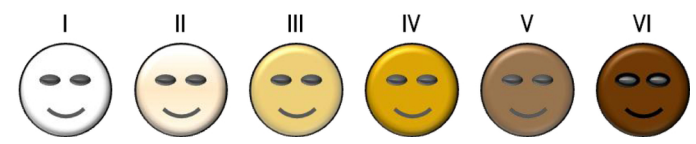

Fig. 3: Fitzpatrick Skin Tone Scale [36]

\section{B. Experimental Configuration}

Our goal is to evaluate a video-based system for monitoring pain on a Raspberry. We selected three hardware configurations for the experiments: one laptop, one PC desktop, and one of the best and most current Raspberry Pi configurations. Comparing the results of both configurations it is possible to analyze the efficiency and effectiveness obtained with Raspeberry. The selected hardware configurations are as follows:

1) Raspberry Pi 4 Model B 64 bits, quad-core, 1,5 GHz. 8GB Memory. Raspberry Pi OS operating system;

2) Laptop Acer i5 $2.40 \mathrm{GHz}$. 8GB DDR4 Memory. GPU GeForce GTX 1650 4GB. Linux Mint operating system; and

3) PC Desktop i7 3770 3.4GHz. 16GB DDR3 Memory. GPU GeForce RTX 2060 SC Gaming - 6GB, GDDR6. Windows 10 operating system.

To capture participants' images, traditional low-cost webcams were used on laptop and PC desktop. For the Raspebbery we used a mini camera developed by the same company that produces the Raspberry: a V2 camera with 8-megapixel resolution. Figure 4 shows the Case for Raspberry/mini camera.

We train and run a RNN model to detect the PSPI value for each face on the video. As presented in Subsection, III-C. it was not possible to train the models on the Raspberry Pi. For training, $70 \%$ of the UNBC-McMaster database was used. The other $30 \%$ of the images were used as a test set.

For the experiments performed for automatic HR measurement, we implement the methods presented in Subsection II-B 


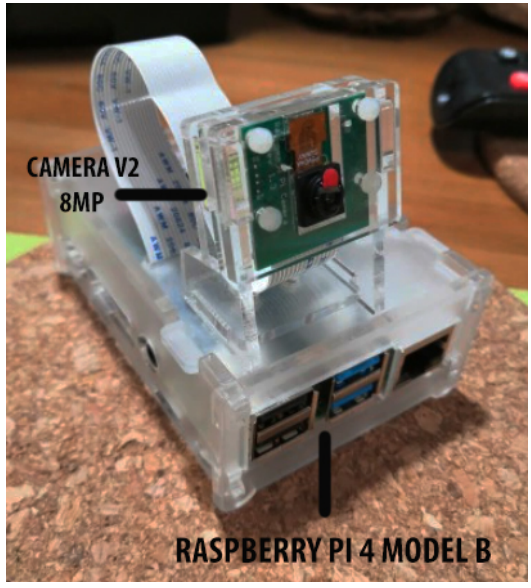

Fig. 4: Case for Raspberry and camera module.

with the Python programming language, using the Dlib and Opencv libraries [30]. This software was executed in real-time with the videos collected in our database.

\section{Experimental Results}

Our experimental results were divided into two groups presented in the follow ordering: i) First will present the results obtained with the prediction of the PSPI value - Equation 1 . and ii) we present the results regarding the HR estimate.

\section{Pain Detection based on PSPI:}

For this experiment, it was necessary to train a RNN model to predict a PSPI value. For training, $70 \%$ of the UNBCMcMaster database was used. Table 1 presents the performance of each hardware selected for the experiment (Subsection III-B in training process. Each row in this table refers to one of the hardware configurations (identified in the first column). The next four columns refer to the following information: i) CPU consumption; ii) GPU consumption; iii) RAM memory consumption; and iv) training time.

TABLE I: RNN training performance to predict PSPI

\begin{tabular}{lllll}
\hline Hardware & CPU & GPU & RAM Memory & Training Time \\
\hline Raspberry Pi & $100 \%$ & not used $^{1}$ & $100 \%$ & no training $^{2}$ \\
Laptop & $44 \%$ & $64 \%$ & $33 \%$ & 73 minutes \\
PC Desktop & $31 \%$ & $36 \%$ & $30 \%$ & 40 minutes \\
\hline \multicolumn{4}{c}{ 2 Raspberry Pi was unable to train the model without restarting due to } \\
& \multicolumn{4}{c}{ overheating }
\end{tabular}

We also evaluated the results of the trained model applied to the test set (30\% of the UNBC-McMaster database) in terms of time and precision. On both hardware, the classification task execution time was very close. About the precision, on all hardware the same result was obtained: a Mean Square Error [37] of 0.23.

HR estimation: The method for HR detection (Subsection II-B) was evaluated in two perspectives: i) hardware resources consumed; and ii) the percentage of divergence between the HR measured by the oximeter and by the rPPG method.
Table II presents three hardware resources consumed to HR measurement: i) CPU consumption; ii) GPU consumption; and iii) RAM memory consumption. It is important to note that in the tests performed with the Raspberry Pi 4 Model B, it was only possible to analyze a minute of video - after that time, the hardware tends to heat up and restart itself.

TABLE II: Resources consumed to HR mensurement

\begin{tabular}{llll}
\hline Hardware & CPU & GPU & RAM memory \\
\hline Raspberry Pi & $33 \%$ & not used & $6 \%$ \\
Laptop & $20 \%$ & $64 \%$ & $6 \%$ \\
PC Desktop & $8 \%$ & $36 \%$ & $4 \%$ \\
\hline
\end{tabular}

Table III presents the percentage of divergence between the HR values achieved with the rPPG and the oximeter. Each hardware configuration used in the experiments is in one table row.

TABLE III: Divergence between Oximeter and rPPG

\begin{tabular}{ll}
\hline Hardware & Percentage of Divergence \\
\hline Raspberry Pi 4 Model B & $4,5 \%$ \\
Laptop & $3,8 \%$ \\
PC Desktop & $3,8 \%$ \\
\hline
\end{tabular}

\section{Analysis of Results}

About the pain detection based on PSPI, is possible to conclude that it is impracticable to train neural network models for that task in a Raspberry PI 4 Model B hardware, as it reaches $100 \%$ of its processing capacity and automatically shuts down as a safety measure to avoid damage overheating the device. This is not a major issue as the model can be trained on other hardware.

Regarding the PSPI classification step, the performance was the same on all hardware used in the case study. In this context, it is viable to monitor pain with the intensity in Raspberry Pi 4 Model B.

About the HR estimation, the percentage of divergence obtained between the results obtained with the rPPG method on the Raspberry Pi 4 Model B in relation to the oximeter is a little higher than the percentage of divergence obtained with the other computers. This performance difference is due to the resolution of the analyzed video - in the Raspberry Pi the standard camera module was used, which has a lower resolution/image quality than the other cameras used in the other computers.

It is possible to purchase cameras of higher quality for use in Raspberry, but the cost of these equipment increases considerably and in addition, with higher quality images, a greater amount of processing would be required, which would impact the device if it restarted more quickly to avoid the superheating.

It should be noted that even though in Raspberry Pi the divergence between Oximeter and the rPPG method is worse 
than that obtained in other devices, the value of $4.5 \%$ is close to the studies found in the state of the art.

In the end, we concluded that Raspberry $\mathrm{Pi}$ is feasible for a video-based pain monitoring system based on the PSPI equation, with very similar results in both hardware. If we combine the approach based on facial expressions with the analyzed algorithm to estimate HR, the result will not be satisfactory for monitoring pain over long periods, as the device will restart to avoid warming up. Future work can be conducted to overcome this limitation.

\section{CONCLUSION}

In this work, the case study was conducted to analyze the efficiency and effectiveness of a non-invasive video-based system for pain assessment performed on the Raspberry Pi. The analyzed system aims to continuously detect two pieces of information from the person's faces: i) HR - with an rPPG method based on Eulerian Video Magnification; and pain intensity, recognized with the PSPI equation - which is detected by an RNN.

Our results indicate that it is feasible to use the Raspberry Pi to monitor pain intensity with the PSPI equation, but it is not feasible to monitor the HR with the analyzed rPPPG method. If the video-based detection of these two multimodal features (with the algorithms analyzed in this case study) it was combined in a Raspberry $\mathrm{Pi}$, it would be unfeasible to use of the pain monitoring system, because of the processing volume, the device will restart to avoid warming up.

We hope that our study will contribute to the development of new algorithms that consume less computational resources and enable the use of the analyzed pain monitoring approach on hardware with features similar to Raspberry. We hope to have also encouraged the development of other applications for low-cost hardware, such as the detection of diseases [38], [39].

\section{REFERENCES}

[1] Thefreedictionary, "Online dictionary," https://www.thefreedictionary. com 2021, [Online; accessed 15-Sep-2021].

[2] M. Benjamin and K. CB, "Miller-keane encyclopedia and dictionary of medicine," Nursing and Allied Health. Philadelphia: Saunders, 1997.

[3] S. N. Raja, D. B. Carr, M. Cohen, N. B. Finnerup, H. Flor, S. Gibson, F. J. Keefe, J. S. Mogil, M. Ringkamp, K. A. Sluka et al., "The revised international association for the study of pain definition of pain: concepts, challenges, and compromises," Pain, vol. 161, no. 9, pp. 19761982, 2020.

[4] N. Katz, "The impact of pain management on quality of life," Journal of pain and symptom management, vol. 24, no. 1, pp. S38-S47, 2002.

[5] A. B. Amspoker, A. L. Snow, B. N. Renn, P. Block, S. Pickens, R. O. Morgan, and M. E. Kunik, "Patient versus informal caregiver proxy reports of pain interference in persons with dementia," Journal of Applied Gerontology, vol. 40, no. 4, pp. 414-422, 2021.

[6] L. P. Carlini, T. M. Heideirich, R. C. Balda, M. C. Barros, R. Guinsburg, and C. E. Thomaz, "Visual perception of pain in neonatal face images," in Workshop de Visão Computacional. SBC, 2019, pp. 37-42.

[7] L. Buzuti, T. Heideirich, M. Barros, R. Guinsburg, and C. Thomaz, "Neonatal pain assessment from facial expression using deep neural networks," in Workshop de Visão Computacional, 2020, pp. 87-92.

[8] K. Herr, P. J. Coyne, E. Ely, C. Gélinas, and R. C. Manworren, "Pain assessment in the patient unable to self-report: clinical practice recommendations in support of the aspmn 2019 position statement," Pain Management Nursing, vol. 20, no. 5, pp. 404-417, 2019.
[9] J. O. Egede, S. Song, T. A. Olugbade, C. Wang, C. D. C. Amanda, H. Meng, M. Aung, N. D. Lane, M. Valstar, and N. Bianchi-Berthouze, "Emopain challenge 2020: Multimodal pain evaluation from facial and bodily expressions," in IEEE International Conference on Automatic Face and Gesture Recognition, 2020, pp. 849-856.

[10] D. Lopez-Martinez and R. Picard, "Multi-task neural networks for personalized pain recognition from physiological signals," in International Conference on Affective Computing and Intelligent Interaction Workshops and Demos, 2017, pp. 181-184.

[11] L. A. da Luz, M. S. A. de Azevedo, V. M. dos Santos, L. A. Reale, F. S. Andrade, K. R. M. Brito, and J. L. dos Santos, "Pênfigo vulgar em homem jovem: relato de caso e revisão da literatura," Brasília Med, vol. 50, no. 4, pp. 346-353, 2013.

[12] P. Shi, V. A. Peris, A. Echiadis, J. Zheng, Y.-S. Zhu, P. Y. S. Cheang, and S.-J. Hu, "Non-contact reflection photoplethysmography towards effective human physiological monitoring," Journal of Medical and Biological Engineering, vol. 30, no. 3, pp. 161-167, 2009.

[13] M. Bonvento, "Acessos vasculares e infecção relacionada à cateter," Revista Brasileira de terapia intensiva, vol. 19, no. 2, pp. 226-230, 2007.

[14] P. S. Addison, D. Jacquel, D. M. Foo, A. Antunes, and U. R. Borg, "Video-based physiologic monitoring during an acute hypoxic challenge: heart rate, respiratory rate, and oxygen saturation," Anesthesia \& Analgesia, vol. 125, no. 3, pp. 860-873, 2017.

[15] D. Fokam and C. Lehmann, "Clinical assessment of arthritic knee pain by infrared thermography," Journal of basic and clinical physiology and pharmacology, vol. 30, no. 3, 2019.

[16] L. I. Castillo, M. E. Browne, T. Hadjistavropoulos, K. M. Prkachin, and R. Goubran, "Automated vs. manual pain coding and heart rate estimations based on videos of older adults with and without dementia," Journal of Rehabilitation and Assistive Technologies Engineering, vol. 7, p. 2055668320950196, 2020.

[17] X. Chen, J. Cheng, R. Song, Y. Liu, R. Ward, and Z. J. Wang, "Videobased heart rate measurement: Recent advances and future prospects," IEEE Transactions on Instrumentation and Measurement, vol. 68, no. 10, pp. 3600-3615, 2018.

[18] K. M. Prkachin and P. E. Solomon, "The structure, reliability and validity of pain expression: Evidence from patients with shoulder pain," Pain, vol. 139, no. 2, pp. 267-274, 2008.

[19] P. Ekman, W. V. Friesen, and J. C. Hager, "Facial action coding system: The manual on cd rom," A Human Face, Salt Lake City, 2002.

[20] S. Hinduja, S. Canavan, and G. Kaur, "Multimodal fusion of physiological signals and facial action units for pain recognition," in IEEE International Conference on Automatic Face and Gesture Recognition. IEEE, 2020, pp. 577-581.

[21] P. Lucey, J. F. Cohn, K. M. Prkachin, P. E. Solomon, and I. Matthews, "Painful data: The unbc-mcmaster shoulder pain expression archive database," in IEEE International Conference on Automatic Face \& Gesture Recognition, 2011, pp. 57-64.

[22] A. B. Hertzman and J. B. Dillon, "Applications of photoelectric plethysmography in peripheral vascular disease," American Heart Journal, vol. 20 , no. 6 , pp. 750-761, 1940.

[23] M. A. Hassan, A. S. Malik, D. Fofi, N. Saad, B. Karasfi, Y. S. Ali, and F. Meriaudeau, "Heart rate estimation using facial video: A review," Biomedical Signal Processing and Control, vol. 38, pp. 346-360, 2017.

[24] H.-Y. Wu, M. Rubinstein, E. Shih, J. Guttag, F. Durand, and W. Freeman, "Eulerian video magnification for revealing subtle changes in the world," ACM transactions on graphics (TOG), vol. 31, no. 4, pp. 1-8, 2012.

[25] S. L. Bennett, R. Goubran, and F. Knoefel, "Adaptive eulerian video magnification methods to extract heart rate from thermal video," in IEEE International Symposium on medical measurements and applications. IEEE, 2016, pp. 1-5.

[26] Y. S. Dosso, A. Bekele, and J. R. Green, "Eulerian magnification of multi-modal rgb-d video for heart rate estimation," in International Symposium on Medical Measurements and Applications. IEEE, 2018, pp. 1-6.

[27] E. Moya-Albor, J. Brieva, H. Ponce, and L. Martínez-Villaseñor, "A non-contact heart rate estimation method using video magnification and neural networks," IEEE Instrumentation \& Measurement Magazine, vol. 23, no. 4, pp. 56-62, 2020.

[28] A. Alzahrani, J. Hosseinkhani, S. Rajan, and E. Ukwatta, "Reducing motion impact on video magnification using wavelet transform and principal component analysis for heart rate estimation," in IEEE International 
Instrumentation and Measurement Technology Conference. IEEE, 2021, pp. 1-6.

[29] A. Alarifi, A. Tolba, and A. S. Hassanein, "Visualization process assisted by the eulerian video magnification algorithm for a heart rate monitoring system: mobile applications," Multimedia Tools and Applications, vol. 79, no. 7, pp. 5149-5160, 2020.

[30] N. Boyko, O. Basystiuk, and N. Shakhovska, "Performance evaluation and comparison of software for face recognition, based on dlib and opencv library," in IEEE Second International Conference on Data Stream Mining \& Processing. IEEE, 2018, pp. 478-482.

[31] A. M. Rodríguez and J. R. Castro, "Pulse rate variability analysis by video using face detection and tracking algorithms," in Annual international conference of the ieee engineering in medicine and biology society. IEEE, 2015, pp. 5696-5699.

[32] E. O. Brigham and R. Morrow, "The fast fourier transform," IEEE spectrum, vol. 4, no. 12, pp. 63-70, 1967.

[33] C. A. Corneanu, M. O. Simón, J. F. Cohn, and S. E. Guerrero, "Survey on RGB, 3D, thermal, and multimodal approaches for facial expression recognition: History, trends, and affect-related applications," IEEE Transactions on Pattern Analysis and Machine Intelligence, vol. 38, no. 8, pp. 1548-1568, 2016.

[34] B. Farnsworth, "Facial action coding system (facs) — a visual guidebook," https://imotions.com/blog/facial-action-coding-system/, 2016.

[35] A. Semwal and N. D. Londhe, "Computer aided pain detection and intensity estimation using compact cnn based fusion network," Applied Soft Computing, vol. 112, p. 107780, 2021.

[36] T. B. Fitzpatrick, "The validity and practicality of sun-reactive skin types i through vi," Archives of dermatology, vol. 124, no. 6, pp. 869-871, 1988.

[37] C.-Y. Chi, C.-H. Chen, C.-C. Feng, and C.-Y. Chen, "Fundamentals of statistical signal processing," Blind Equalization and System Identification: Batch Processing Algorithms, Performance and Applications, pp. 83-182, 2006.

[38] J. Souza, T. de Oliveira, C. Casa, and A. Ortoncelli, "Automatic detection of lupus butterfly malar rash based on transfer learning," in Workshop de Visão Computacional. SBC, 2020, pp. 36-40.

[39] Í. Gama, A. Coelho, and M. Baffa, "Fundus eye images classification for diabetic retinopathy detection using very deep convolutional neural network," in Workshop de Visão Computacional. SBC, 2020, pp. 24-29. 